\title{
Primary inoculation tuberculosis: a report of a rare entity
}

\author{
Fatma Sule Afsar ${ }^{[1]}$, Sinan Ozcelik ${ }^{[1]}$, Sila Seremet Uysal ${ }^{[1]}$, \\ Murat Ermete $^{[2]}$ and Ilhan Afsar ${ }^{[3]}$
}

[1]. Department of Dermatology, Ataturk Research and Training Hospital, Izmir, Turkey. [2]. Department of Pathology, Ataturk Research and Training Hospital, Izmir, Turkey. [3]. Department of Medical Microbiology, Ataturk Research and Training Hospital, Izmir, Turkey.

\begin{abstract}
Primary inoculation tuberculosis is an exogenous infection resulting from direct inoculation of bacteria into individuals with no acquired immunity to the organism. We report a 63-year-old male patient who was diagnosed with primary inoculation tuberculosis on the basis of clinical appearance and histopathological examination. The findings from this case emphasize the importance of clinical and histopathological findings in this rarely seen form of skin tuberculosis if the organism cannot be shown to grow in culture.
\end{abstract}

Keywords: Diagnosis. Skin. Tuberculosis.

\section{INTRODUCTION}

Cutaneous tuberculosis describes dermatological manifestations of tuberculosis involving the skin, which can be caused by Mycobacterium tuberculosis, Mycobacterium bovis, and the bacillus Calmette-Guérin vaccine, and accounts for $1.5 \%$ of extrapulmonary cases ${ }^{(1)(2)}$. Classification has been attempted according to morphology and the mode of infection or immunological state of the host ${ }^{(3)}$.

Primary inoculation tuberculosis results from direct inoculation of Mycobacterium tuberculosis into the skin of a person who has no natural or artificially acquired immunity against the organism. The pathogenesis requires a break in the skin due to an abrasion or injury, which allows entry of the tubercle ${ }^{(4)}$. Within two to four weeks, an inflammatory papule develops at the inoculation site and evolves into a firm, shallow, nontender, nonhealing, undermined ulcer with a granulomatous base. Painless, regional lymphadenopathy is frequently apparent around the time at which the tuberculin skin test (TST) becomes positive ${ }^{(1)}$. We report a case of primary inoculation tuberculosis, which is rarely observed.

\section{CASE REPORT}

A 63-year-old male patient presented with an elevated, crusted wound on his lower right leg and a painless lump in his right groin. Physical examination revealed a violaceous, ulcerated, and crusted nodule $(3 \times 3 \mathrm{~cm})$, which had appeared

\footnotetext{
Address to: Dr. Fatma Sule Afsar. 2040 Sok. Kugu 122 D:50 35540 Mavisehir Karsiyaka Izmir, Turkey.

Phone: 90532 295-8536

e-mail: suleafsar@hotmail.com

Received 4 December 2014

Accepted 4 February 2015
}

in the right pretibial area 2 months previously, and a soft, fixed, palpable lymphadenopathy, which had been present for 4 weeks. The physical examination was otherwise normal.

The patient was not aware of trauma at the lesion site, and his family history was unremarkable. He reported a history of left adrenalectomy and chemotherapy for adrenocortical adenoma and liposarcoma 6 years previously.

Results of a full blood count, routine biochemistry analysis, erythrocyte sedimentation rate test, chest radiography, and urine sample for microscopic examination were all normal, and antihuman immunodeficiency virus and venereal disease research laboratory tests results were negative. Complete abdominal ultrasonography findings were normal, with no evidence of lymph node involvement. The TST result was negative.

The report on the biopsy of the lesion described nonspecific granulation tissue; therefore, a second biopsy was performed, with prediagnoses of cutaneous tuberculosis, cutaneous leishmaniasis, syphilis, and sporotrichosis. The tissue sections tested negative for acid-fast-bacilli using the Ehrlich-ZiehlNeelsen stain but revealed granuloma formation consisting of mononuclear inflammatory cells, epithelioid histiocytes, and Langhans-type giant cells (Figure 1), which was reported as cutaneous tuberculosis. Soft tissue ultrasonography of the patient's right inguinal region identified a regular contoured lymph node $(1 \times 3 \mathrm{~cm})$ with preserved fatty hilum.

The patient had no history of contact with, or vaccination against, tuberculosis prior to the appearance of the lesion. When the TST was repeated, an induration of $30 \mathrm{~mm}$ was observed, and the culture on Löwenstein-Jensen medium was negative. The BACTEC MGIT system result was also negative for mycobacterial recovery.

Adiagnosis of primary inoculation tuberculosis was established according to the patient's history, physical examination, conversion of TST from negative to positive, and histopathological findings. The patient was treated at the Department of Pulmonary Medicine, 

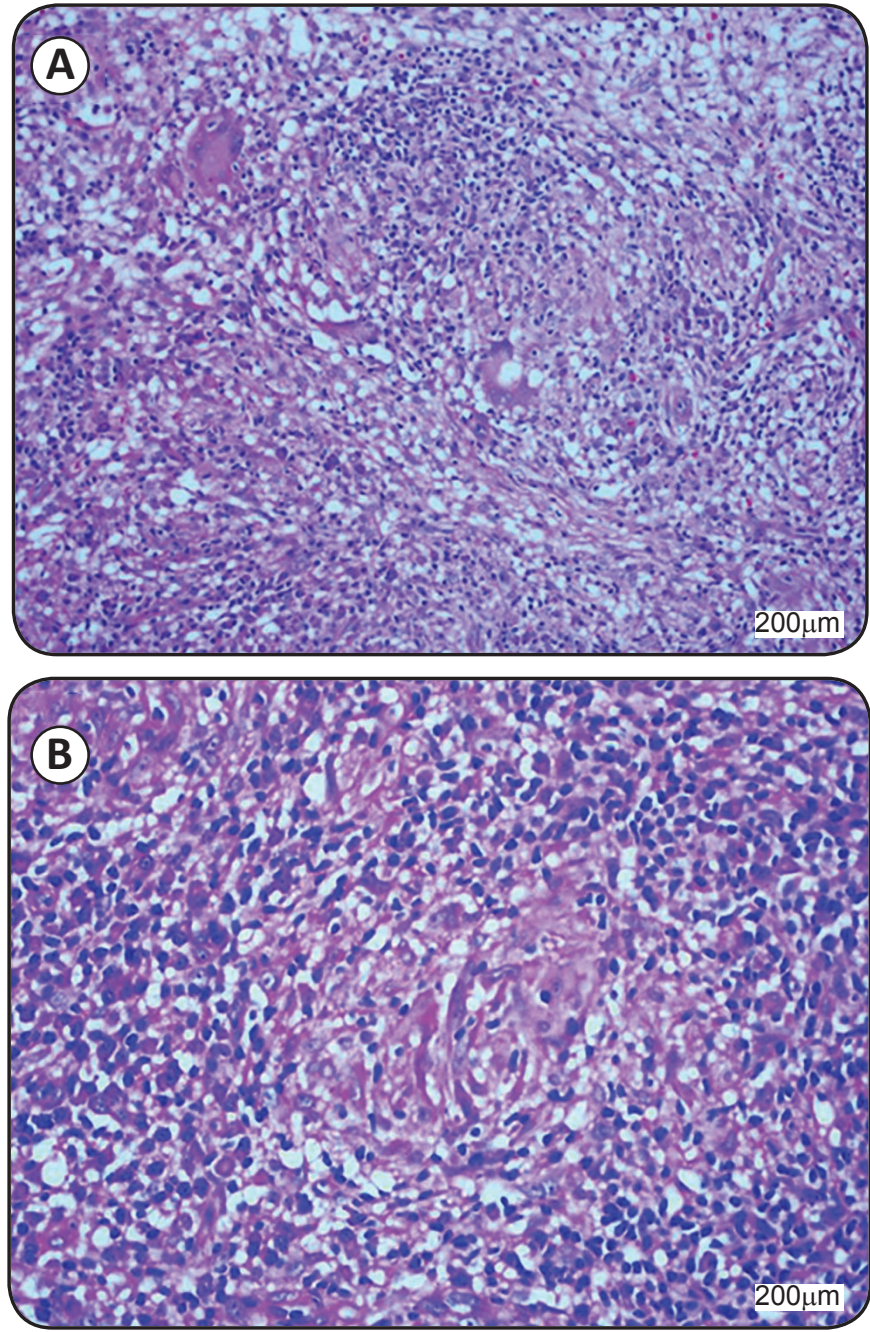

FIGURE 1 - A) Granuloma formation consisting of mononuclear cells, epithelioid histiocytes, and Langhans-type giant cells (hematoxylin-eosin $\times 20$ ). B) Magnified histopathological appearance of the granuloma formation (hematoxylin-eosin $\times 40$ ).

and isoniazid $300 \mathrm{mg} /$ day, rifampicin $600 \mathrm{mg} /$ day, ethambutol $1,500 \mathrm{mg} /$ day, and morphozinamide $2,000 \mathrm{mg} /$ day were prescribed for the first 2 months, with isoniazid and rifampicin prescribed for 5 months thereafter. During his treatment, full blood count, erythrocyte sedimentation rate, and liver and kidney function were evaluated every 4 weeks, and the results were within normal limits. The violaceous and crusted nodule on the right pretibial area showed a remarkable improvement within 4 months (Figure 2) and had almost healed 7 months subsequent to antituberculous treatment.

\section{DIscussion}

Tuberculosis is one of the most prevalent infectious diseases in developing countries. Clinically, tuberculosis can present as pulmonary or extrapulmonary disease. Extrapulmonary involvement can be observed at every site. Cutaneous infection is rare and accounts for $0.1 \%$ of consultations in dermatology

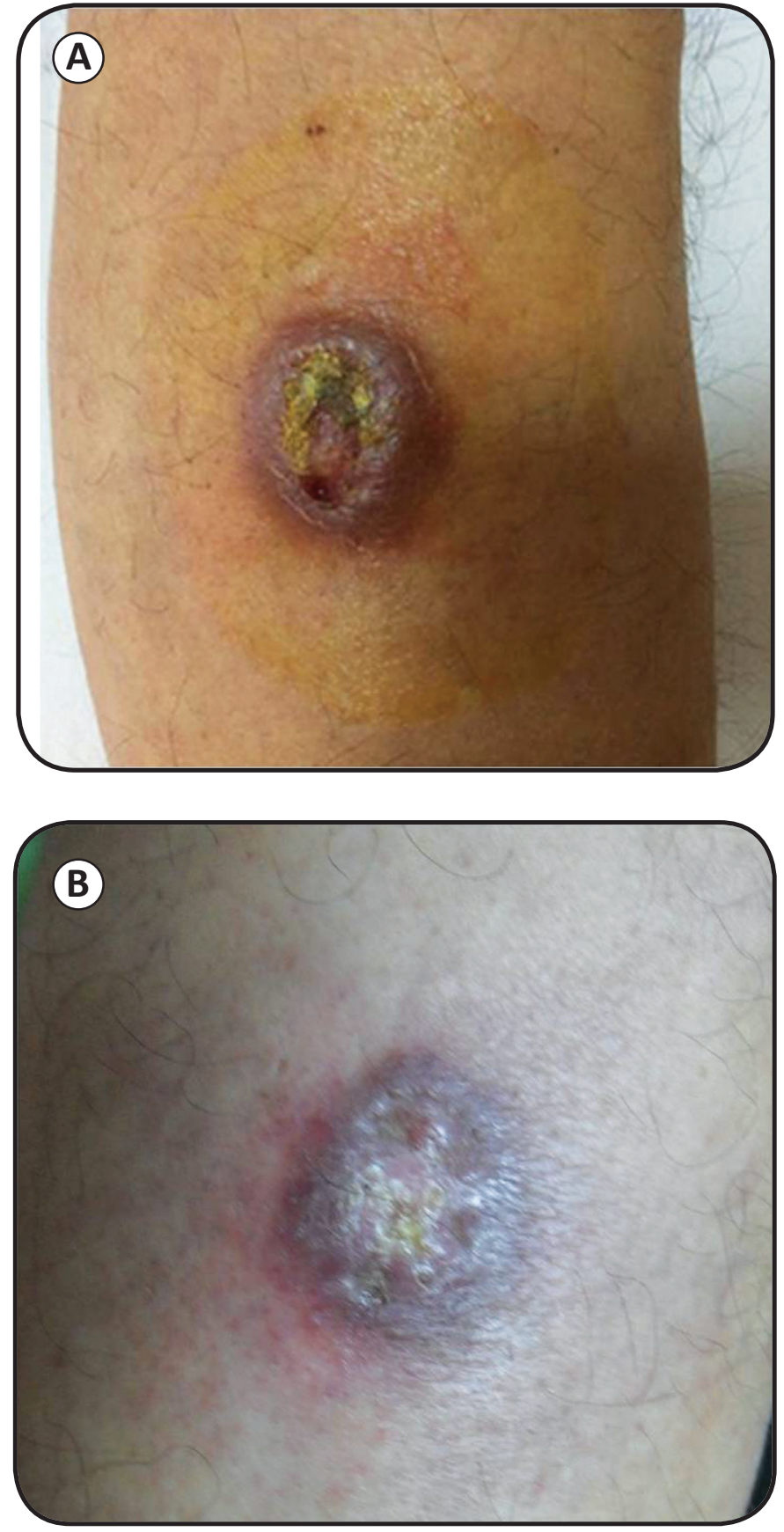

FIGURE 2 - A) Ulcerated and crusted nodule in the right pretibial area. B) Remarkable improvement observed after 4 months of antituberculous treatment.

practice ${ }^{(5)}$. Clinical manifestation of cutaneous tuberculosis is so variable that a high index of suspicion is required for diagnosis. Cutaneous tuberculosis is classified according to the source of the skin infection. Endogenous infection occurs in a host previously infected with Mycobacterium tuberculosis via contiguous hematogenous or lymphatic extension, and exogenous infection results from direct inoculation of bacteria into the $\operatorname{skin}^{(6)}$.

Primary inoculation tuberculosis requires a break in the normal barrier function of the skin of a person with no natural or 
acquired immunity against the organism ${ }^{(4)}$ (7). It usually occurs, often unnoticed, through minor abrasions or injury. It has also been reported to occur following tattooing, circumcision, earpiercing, intramuscular injection, and tooth extraction ${ }^{(7)}$. We did not determine a primary focus with respect to the cause of hematogenous or lymphatic spread in our patient and suspected that he had sustained an unnoticed minor trauma to the lesion site.

Primary inoculation tuberculosis lesions generally appear 2-4 weeks subsequent to inoculation and present as erythematous painless papules or nodules ${ }^{(4)}$. Histologically, early changes consisting of acute neutrophilic inflammation with necrosis are present prior to progression to granuloma formation and caseation. Bacilli might be observed in the early weeks of infection ${ }^{(2)}$. Painless regional lymphadenopathy develops three to eight weeks subsequent to infection, and a tuberculoid appearance and caseation necrosis may be observed in histopathology(4). The multibacillary lesion becomes paucibacillary as host immunity develops, and the TST becomes positive ${ }^{(1)(3)}$. Our patient had a typical nodular lesion and regional lymphadenopathy, which developed 4 weeks subsequent to the infection. The TST result, which was initially negative, became strongly positive, but the bacilli were not observed in tissue sections using the Ehrlich-Ziehl-Neelsen stain, although granuloma formation was present.

Differential diagnoses considered in primary inoculation tuberculosis include foreign body granuloma, sarcoidosis, tertiary syphilis, and infection with other mycobacteria. Caseation necrosis is not typically observed in granulomas secondary to foreign bodies and sarcoidosis ${ }^{(7)}$. However, absence of caseation necrosis does not necessarily rule out diagnosis of cutaneous tuberculosis ${ }^{(8)(9)}$. Periodic acid-Schiff stain and fungal culture can be used in the differential diagnosis of sporotrichosis. Syphilis can be excluded via a serological test and shows vascular changes and predominant plasma cell infiltration in histology ${ }^{(4)}$. Atypical mycobacteria that commonly cause skin infections are diverse in clinical presentation and geographic prevalence. The most common atypical mycobacterial skin infection, with Mycobacterium marinum, is more prevalent in immunocompromised hosts and often characterized by multiple violaceous papules or nodules, which may present in a sporotrichoid pattern ${ }^{(10)}$. Atypical mycobacterial infections are not prevalent in our geographic region. In addition, the patient described herein was immunocompetent, while his clinical presentation was not suggestive of characteristic lesions of atypical mycobacterial infection. Polymerase chain reaction enzyme-linked immunosorbent assay and radioimmunoassay are new but expensive techniques used in the diagnosis of tuberculosis and are not considered routine investigations ${ }^{(7)(11)}$.

Primary inoculation tuberculosis usually heals within one year; however, acute miliary tuberculosis can develop in patients with poor immunity. In addition, latent foci of infection can later reactivate locally or shed organism heterogeneously, and lupus vulgaris or tuberculosis verrucosa cutis may develop. Regional lymph nodes may liquefy, producing scrofuloderma ${ }^{(12)}$.

Multiple antituberculous agents should be administered for a period of no less than 6 months ${ }^{(6)}$. Combination treatment, including isoniazid, rifampicin, ethambutol, and morphozinamide, for the first 2 months, with isoniazid and rifampicin for 5 months thereafter, was well tolerated by our patient, and a remarkable improvement was achieved. Cutaneous tuberculosis can be difficult to confirm, and on occasion, a diagnosis is only established retrospectively, following response to a therapeutic trial ${ }^{(5)}$.

In conclusion, careful consideration of clinical presentation is very important in cutaneous tuberculosis. Therefore, it should be considered in patients with suspicious skin lesions which have histopathological correlation, TST reaction, and response to antituberculous treatment when bacilli cannot be demonstrated in tissue sections or culture.

\section{REFERENCES}

1. Frankel A, Penrose C, Emer J. Cutaneous tuberculosis: a practical case report and review for the dermatologist. J Clin Aesthet Dermatol 2009; 2:19-27.

2. Gawkrodger DJ. Mycobacterial infections. In: Champion RH, Burton JL, Burns DA, Breathnach SM, editors. Rook Textbook of Dermatology. $6^{\text {th }}$ ed. Oxford: Blackwell Science; 1998. p. 1187-1206.

3. Tappainer G, Wolff K. Tuberculosis and other mycobacterial infections. In: Freedberg IM, Eisen AZ, Wolff K, Austen KF, Goldsmith LA, Katz SI, et al, editors. Dermatology in General Medicine. $5^{\text {th }}$ ed. New York: McGraw Hill; 1999. p. 2274-2292.

4. Kim JK, Kim TY, Kim DH, Yoon MS. Three cases of primary inoculation tuberculosis as a result of illegal acupuncture. Ann Dermatol 2010; 22:341-345.

5. Ho CK, Ho MH, Chong LY. Cutaneous tuberculosis in Hong Kong: an update. Hong Kong Med J 2006; 12:272-277.

6. Tapias L, Tapias-Vargas LF, Tapias-Vargas L. Primary cutaneous inoculation tuberculosis in a healthcare worker as a result of a surgical accident. Int J Dermatol 2008; 47:833-835.

7. Wong HW, Tay YK, Sim CS. Papular eruption on a tattoo: a case of primary inoculation tuberculosis. Australas J Dermatol 2005; 46:84-87.

8. Thomas S, Suhas S, Pai KM, Raghu AR. Lupus vulgaris-report of a case with facial involvement. Br Dent J 2005; 198:135-137.

9. Sehgal VN, Jain MK, Srivastava G. Changing pattern of cutaneous tuberculosis. A prospective study. Int J Dermatol 1989; 28:231-236.

10. Afzal A, Nadeem M, Aman S, Kazmi AH. Mycobacterium marinum infection: A case report. J Pak Assoc Dermatol 2009; 19:48-51.

11. Jain S, Sehgal VN. Inoculation cutaneous tuberculosis. Australas J Dermatol 1998; 39:183-185.

12. James WD. Disease and the environment. In: Zajchuk R, editor. Textbook of Military Medicine (Military Dermatology). Washington, DC: Borden Institute; 1994. p. 1-617. 